\title{
Synchrotron radiation applications of charge coupled device detectors (invited)
}

Roy Clarke

University of Michigan, Department of Physics, Ann Arbor, Michigan 48109-1120

Walter P. Lowe and R. A. Macharrie

AT\&T Bell Laboratories, Murray Hill, New Jersey 07974-2070

C. Brizard and B. G. Rodricks

Advanced Photon Source, Argonne National Laboratory, Argonne, Illinois 60439

(Presented on 17 July 1991)

Scientific charge coupled devices (CCDs) offer many opportunities for high brightness synchrotron radiation applications where good spatial resolution and fast data acquisition are important. We describe the use of virtual-phase CCD pixel arrays as two-dimensional area detectors illustrating the techniques with results from recent $\mathrm{x}$-ray scattering, imaging, and absorption spectroscopy studies at NSLS, CHESS, SRC, and LURE DCI. The virtual phase architecture allows direct frontside illumination of the CCD detector chips giving advantages in the speed and sensitivity of the detector. Combining developments in $x$ ray optics (dispersive geometry), position sensitive area detectors (CCDs), and fast data acquisition, we have been able to perform time-resolved measurements at the microsecond level. Current developments include faster data transfer rates so that the single bunch timing structure of third generation synchrotron sources can be exploited.

\section{INTRODUCTION}

The advent of high-brightness synchrotron sources has extended $x$-ray research into the time domain. ${ }^{1}$ It is now possible to study real materials under actual operating conditions, e.g., during thermal processing, under high stress or while a structural phase transition is taking place. ${ }^{2}$

While a great deal of effort has been directed towards the $x$-ray source itself, particularly the installation of insertion devices in storage rings, a serious limitation exists with respect to detection and imaging systems. Without a sustained effort in this area it will not be possible to fully exploit the unique advantages of third generation synchrotron sources (viz., high brilliance, coherence, polarization, and fast-pulse characteristics). The operating characteristics of existing imaging systems fall short of what is required as regards speed, energy and spatial resolution, dynamic range, and data handling capabilities.

In this article we report on progress that is being made on one promising type of solid-state array detector, the charge coupled device (CCD). Several prototype detectors have been constructed based on this technology, and a considerable amount of experience has been gained over the past few years in their operation at various synchrotron radiation facilities around the world. In what follows we will illustrate some of these recent studies with examples across a diverse range of synchrotron applications pointing out the strengths and limitations of this particular type of detector. We will conclude with some thoughts about the future prospects for $\mathrm{x}$-ray detector development.

\section{CCD IMAGING AND READOUT}

The design and operation of our CCD area detector has been reported in previous papers. ${ }^{3,4}$ Here we summarize the salient features:

\section{A. CCD pixel array and readout}

The CCDs used in our present detectors are so-called "virtual phase" devices. The primary advantage of this type of architecture for $\mathrm{x}$-ray detection is that the CCD chip can be operated efficiently in frontside illumination

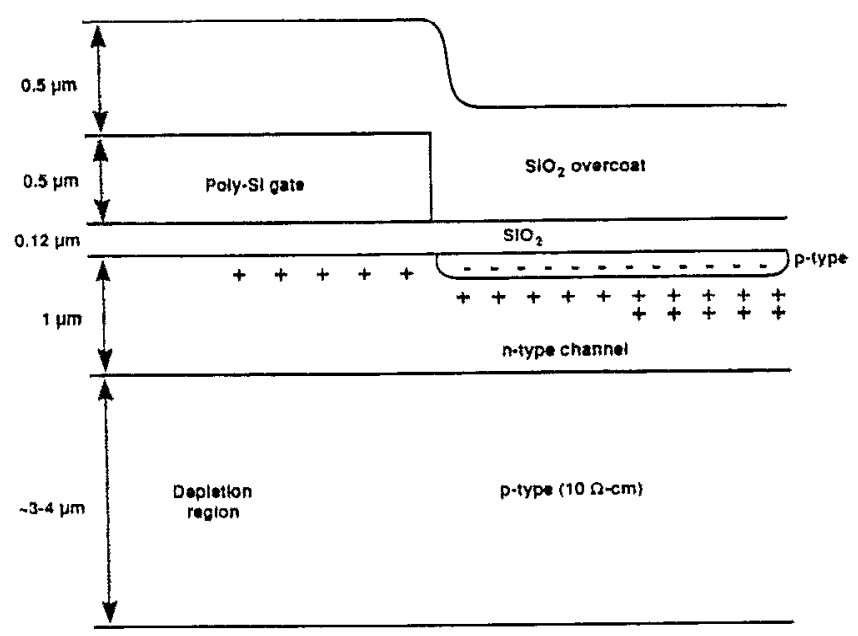

FIG. 1. Schematic of construction of TI4849 CCD detector showing ion-implanted "virtual electrodes." 
TABLE. I. Performance characteristics of virtual phase CCD detector.

\begin{tabular}{ll}
\hline \hline CCD chip & TIJ 215 \\
Frame size (pixels) & $1024 \times 1024$ \\
$\quad$ Pixel size & $12 \mu \mathrm{m} \times 12 \mu \mathrm{m}$ \\
Image size & $17.2 \mathrm{~mm}$ diagonal \\
Noise level $\left(e^{-} /\right.$pixel) & 55 \\
Operating temperature & $-50^{\circ} \mathrm{C}$ \\
Change transfer efficiency & 0.99997 \\
Gain $\left.\mu \mathrm{V} / e^{-}\right)$ & 5.6 \\
Full well depth ( $\left.e^{-}\right)$ & 70000 \\
Uniformity (50\% saturation) & $5 \%$ \\
Linearity & $<0.5 \%$ \\
Data rate & $10 \mathrm{MHz}$ \\
Dynamic range & $10^{3}-10^{4}$ \\
\hline \hline
\end{tabular}

because only $1 / 3$ of the face of the pixel array is covered by readout electrodes. The remaining area is relatively transparent to the incoming radiation since the "virtual " electrodes consist of ion-implanted buried channels. Figure 1 illustrates the actual fabrication in more detail.

An older type of virtual phase chip, the Texas Instruments 4849, has been used extensively for many of our experiments. It has 584 rows of $22.5 \mu \mathrm{m}$ pixels arranged in 390 columns forming an active area of approximately 8 $\times 13 \mathrm{~mm}^{2}$. This is rather small for many applications (especially for crystallographic uses ${ }^{5}$ where large area is desirable); however, area magnification schemes are being explored using either lenses or fiber-optic tapers in conjunction with a phosphor screen. A more recent version of the 4849 chip, the TIJ215, has also been implemented in our group. It is basically identical in construction to the 4849 but has a larger array $(1024 \times 1024)$ with correspondingly smaller pixels $(12 \mu \mathrm{m})$. (See Table I).

Two different readout modes have been adopted so far for synchrotron applications: "full frame" and "streak camera." In the former mode a full frame image is collected on the whole pixel array and the readout is accomplished by transferring the collected charges down the columns of the chip (parallel transfer) into a serial register. Here each pixel charge is read out serially, amplified, digitized, and stored. This last operation is inherently slow $(\sim 2 \mu \mathrm{s} /$ pixel $)$ and presents a serious limitation on speed ( $\sim$ few seconds for full-frame readout).

The streak camera mode of readout is an attempt to achieve much faster data acquisition. The chip is masked so that only one row of pixels is exposed. After a predetermined exposure time the charge accumulated on this row is downshifted by the parallel register. The emptied row is exposed again and its charge downshifted. In this way a sequential record of up to 1000 one-dimensional exposures is stored on the masked rows. Since the data remains on the chip, the time resolution is limited only by the speed of the parallel transfer and not by the digitization step, or the transfer to disc. At present, capacitive effects limit the transfer rate $\sim 100 \mathrm{kHz}$ so that the time resolution is about $10 \mu \mathrm{s}$. There are important reasons to push this limit downwards by an order of magnitude, for example in order to streak data faster than the single bunch period [ $\sim 3 \mu \mathrm{s}$ at the Advanced Photon Source (APS)]. In

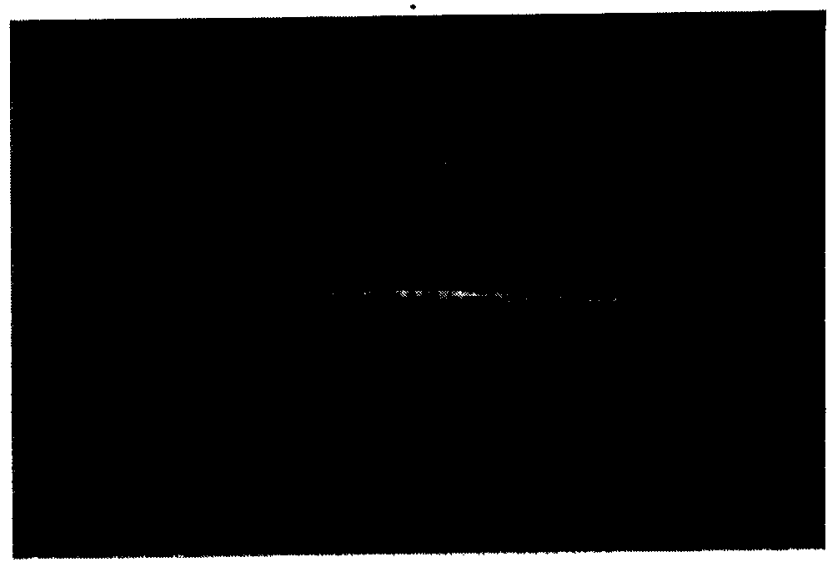

FIG. 2. CCD image of the phase space ellipse of the first harmonic from APS protolype undulator "A." The image was obtained by scanning a 10 $\mu \mathrm{m}$ slit through the beam at a distance of $18.5 \mathrm{~m}$ from the source. The size of the image is approximately $0.25 \mathrm{~mm}$ (vertical) by $2.5 \mathrm{~mm}$ (horizontal) giving a vertical divergence of $\sim 14 \mu \mathrm{rad}$. The undulator was operated at the CHESS facility (Data courtesy of P. J. Viccaro, R. Dejus, B. Lai, B. Rodricks, and W. Yun).

that case one might be able to utilize the pulsed structure of the beam to achieve time resolution at or below the level of the pulse length $(\sim 100$ ps at APS).

\section{B. Virtual phase design and radiation hardness}

A further advantage often claimed for the virtualphase CCD concerns its resistance to radiation damage. ${ }^{6}$ This is important for the direct detection of $x$ rays (for example if the requirement of fast time resolution precludes the use of a phosphor). The deep ion implants for the virtual electrodes supposedly form a more robust pinning framework for the transfer potential, leading to enhanced radiation hardness. We have checked this claim by making careful measurements of the charge transfer efficiency (CTE) and dark current levels as a function of total absorbed dose of $8 \mathrm{keV} x$ rays. We find ${ }^{7}$ that there is no measurable deterioration at a dose of $25 \mathrm{krad}$, which corresponds to about $20 \mathrm{~h}$ of continuous use at the National Synchrotron Light Source (NSLS) on a bending magnet. At doses several times larger than this, a reduction in the CTE onsets. Dark current is nol generally a problem because the detector chips are usually cooled (to $-50^{\circ} \mathrm{C}$ ), but CTE reduction can be very detrimental to the detector sensitivity.

\section{CCD APPLICATIONS IN SYNCHROTRON RADIATION}

The characteristics of scientific CCDs lend themselves to a great variety of experimental applications utilizing the unique characteristics of synchrotron radiation. Studies ranging over $\mathrm{x}$-ray optics evaluation, beam diagnostics, $\mathrm{x}$ ray absorption spectroscopy, photoelectron microscopy, and time-resolved $\mathrm{x}$-ray scattering have all benefitted from the development of CCD $x$-ray detector technology. In this section we give some examples from this work in order to illustrate the interesting possibilities that $\mathrm{CCDs}$ offer for 


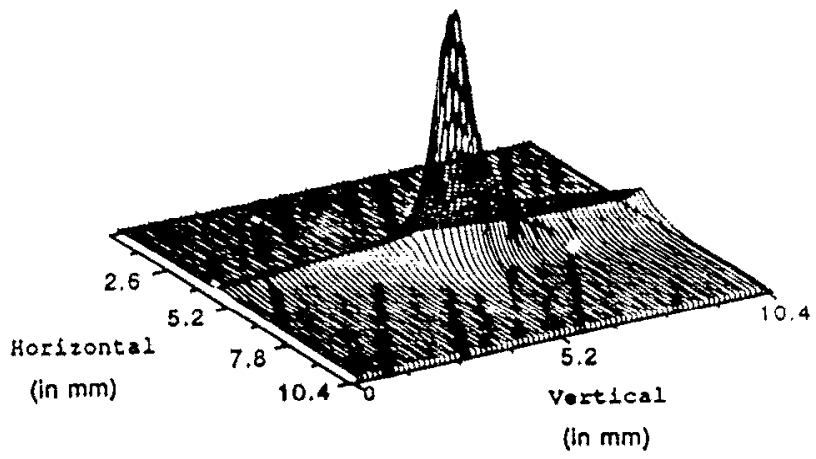

FIG. 3. CCD frame readout of $x$-ray bending magnet radiation reflected from the vertical focusing mirror on X-16B NSLS.

synchrotron science and to emphasize how advantageous area detectors can be for certain kinds of measurements.

\section{A. $\mathrm{X}$-ray optics evaluation}

Characterization of the quality of $x$-ray optics (mirrors, zone plates, monochromator systems, etc.) is becoming increasingly important to a number of applications ranging from lithography to $x$-ray astronomy. A problem of immediate concern to the synchrotron community is how to measure the quality of high brilliance beams from insertion devices such as undulators. Figure 2 illustrates the use of $\mathrm{CCD}$ detectors for imaging the beam from a hard $\mathrm{x}$-ray undulator recently tested at the Cornell High Energy Synchrotron Source (CHESS). The ability to map out the phase space ellipse in this way, quickly and efficiently, provides a valuable tool not only for evaluating the performance of the undulator but also as a diagnostic of the orbit stability and emittance characteristics. Given the extremely high brilliance of such insertion devices it is of equal concern that optical elements inserted into the beam do not introduce significant degradation. The demands on optical figure in this case are very high. A CCD area detector is a very convenient and rapid way to obtain an image from an $\mathrm{x}$-ray mirror for different alignment settings. Figure 3 shows a full frame readout of the $x$-ray beam reflected at grazing incidence from the harmonic rejection mirror on Beamline X16-B. The vertical streak on the leading edge of the main peak arises from a combination of surface roughness and errors in the bending figure of the mirror which is designed to also provide vertical focusing. A further example of CCDs being used to evaluate the quality of an $x$-ray mirror, this time ultimately for $\mathrm{x}$-ray astronomy applications, is shown in Fig. 4. The mirror in qucstion is a double-bounce Wolter-I grazing incidence mirror and is very tedious to align in the synchrotron $x$-ray beam. However, using the CCD imager it was relatively straightforward to obtain a series of images in order to evaluate its performance. Surface roughness of typically $20 \AA$ was demonstrated on gold covered lacquerpolished surfaces. ${ }^{8,9}$

\section{B. SImultaneous extended $x$-ray absorption fine structure (EXAFS) and diffraction on multilayer samples}

Curved-crystal dispersive measurements pioneered many years ago by Cauchois ${ }^{10}$ have proved to be quite versatile for a wide range of different experiments. Fontaine and co-workers ${ }^{11}$ have performed dispersive EXAFS by recording data on a linear position-sensitive detector at the LURE DCI source. In this case the curvature of the crystal [Si(311)] is adjusted to give a large energy spread $(\sim 500 \mathrm{ev})$ in the horizontal fan. Each pixel of the detector records absorption at a particular energy and an EXAFS

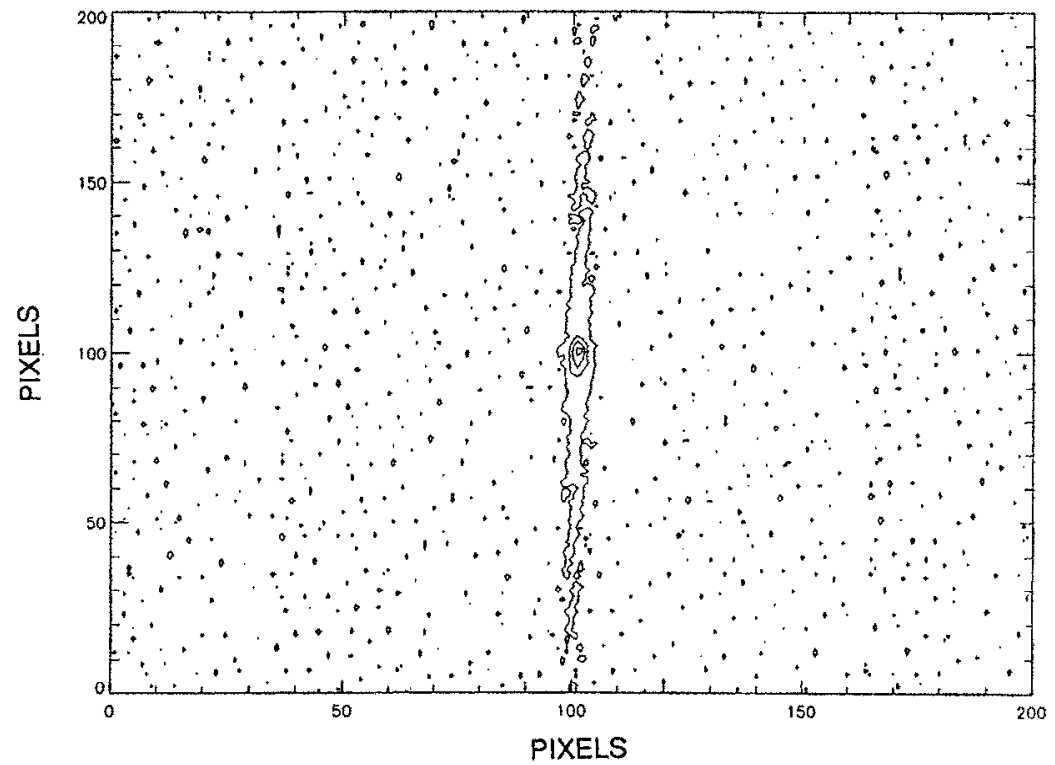

FIG. 4. CCD diagnostic test of $x$-ray mirror performance at $5 \mathrm{keV}$. The surface roughness is calculated from the refiected power in the specular direction (Ref. 8). 


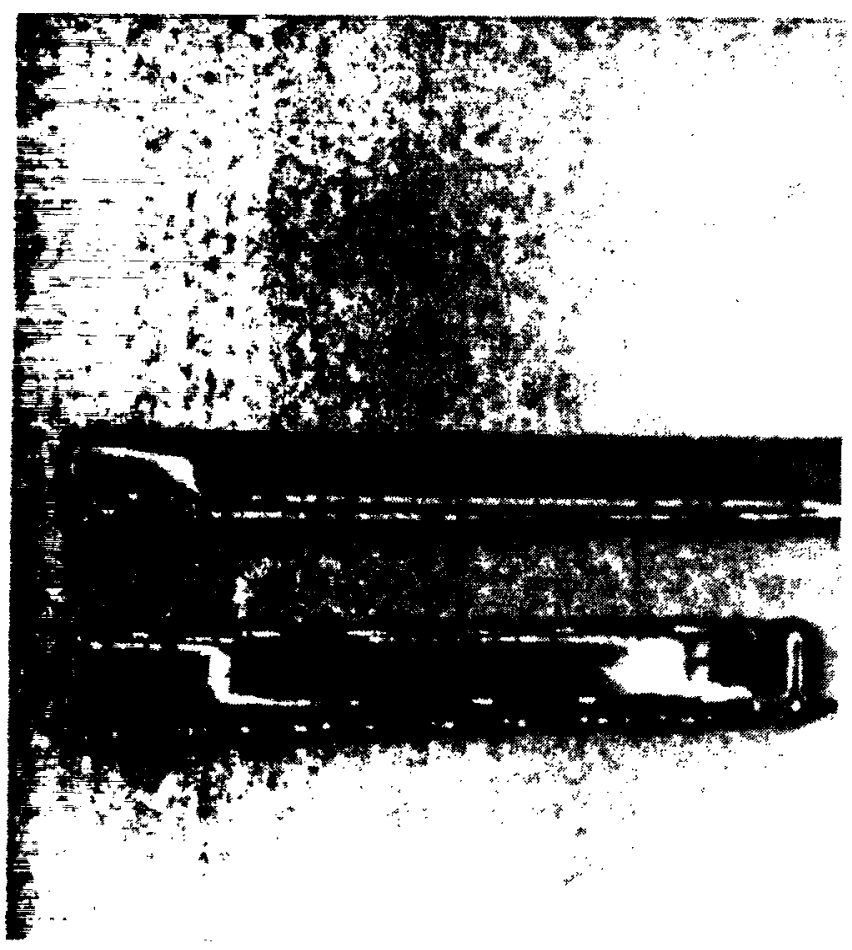

FIG. 5. Dispersive EXAFS data from a Pt-C multilayer recorded on a $\mathrm{CCD}$. The absorption spectrum is measured through the $L_{\mathrm{III}}$ absorption edge of platinum $(E \approx 11.6 \mathrm{keV})$. Shown here is the absorption spectrum for both the specular beam and the first order diffracted beam measured simultaneously on the CCD. The $L_{111}$ edge can be seen clearly as the energy increases along the horizontal axis. (Ref. 12).

spectrum can be recorded in milliseconds without mechanically scanning the energy.

It has also been demonstrated that simultaneous EXAFS and reflectivity studies are possible using a CCD area detector. In this method the dispersive absorption spectra of both specular and first-order diffracted beams from a multilayer sample are recorded together on a CCD area detector in a time frame of $100 \mathrm{~ms}$ (Fig. 5). This measurement takes advantage of the area detection in an interesting way: the energy of the beam varying along the horizontal axis and the momentum transfer in the highly collimated vertical direction. Such data are useful because of the possibility of probing simultaneously the short range order (EXAFS) of a particular element in the multilayer together with the long-range information provided by diffraction.

\section{Photoelectron microscopy}

An interesting recent development in soft x-ray imagining techniques utilizing the high resolution of CCDs is that of photoelectron microscopy. ${ }^{13}$ The surface of a sample is illuminated with monochromatic soft $\mathrm{x}$ rays from the Aladdin Synchrotron at the Synchrotron Radiation Center in Wisconsin. As the energy is scanned through the core levels, photoelectrons are emitted which are then focused electrostatically onto a channel plate to form a magnified image. The CCD records the intensity on a dot-matrix

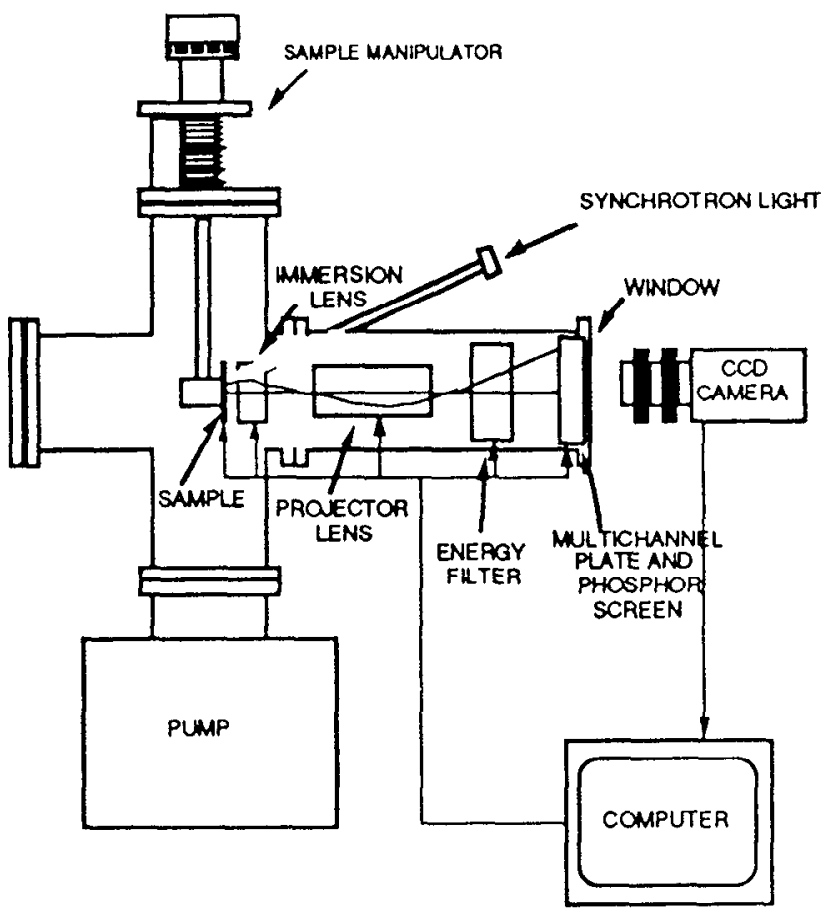

FIG. 6. Photoelectron microscope used with CCD camera to record soft $\mathrm{x}$-ray photoelectron images at the Synchrotron Radiation Center, Wisconsin (Courtesy of J. C. Campuzano, Ref. 13).

phosphor screen behind the channel plate (Fig. 6). The image so formed is a high resolution ( $\sim 600 \AA$ is possible) map of the sample surface containing information on the details of the local bonding and chemical environment of the specific elements that are being scanned. Figure 7 is such an image of a high temperature superconductor showing variations in the oxygen content on a submicron scale. The particular advantage of the CCD detector here is that it can record high-resolution frames of data on a computer

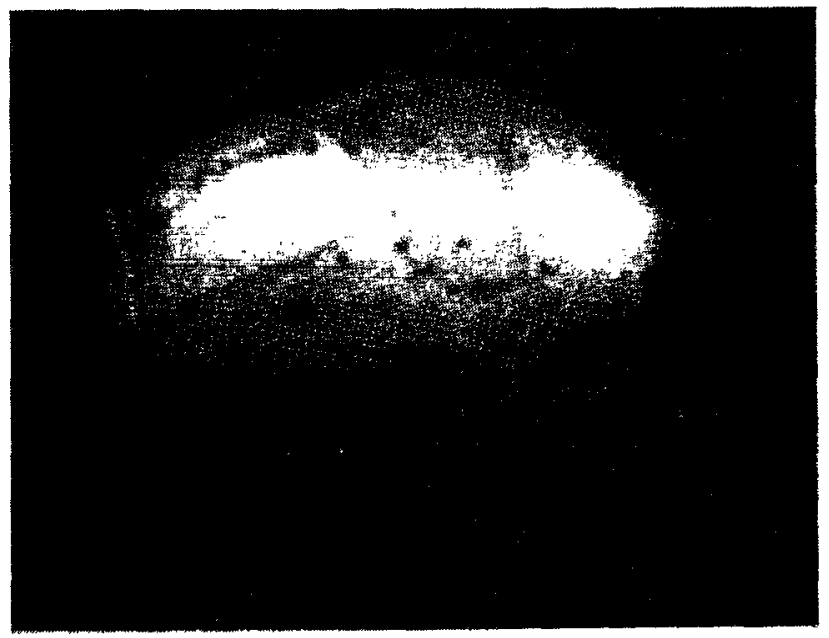

FIG. 7. Photoelectron micrograph of a thallium high temperature superconducting film (Ref. 13). 


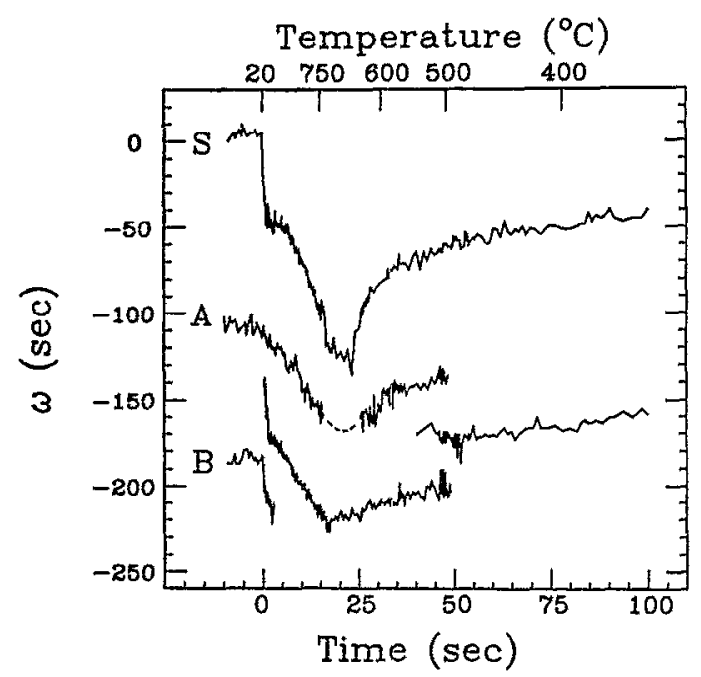

FIG. 8. Rapid thermal annealing of GaAs-InGaAs-GaAs SQW structure. X-ray peak position at $\mathrm{CCD}$ detector is shown as a function of time during rapid thermal annealing. A quartz-halogen heating lamp is turned on at $t=0$ and off at $t=30 \mathrm{~s}$ during which the sample temperature reaches $750^{\circ} \mathrm{C}$ (as measured by thermocouples in front of, and behind, the sample). Curves $A$ and $B$ refer to peaks arising from the interference of $x$ rays scattered from the two GaAs layers of the SQW shifted in phase by interface strains. The peak positions are measured with reference to the initial position $(\omega=0)$ of the substrate peak. Curve $S$ corresponds to scattering from the substrate and unstrained GaAs layers.

at each particular energy, facilitating analysis of large amounts of spectral data.

\section{Real-time $x$-ray scattering}

The above applications utilize the two-dimensional readout of the CCD detector to obtain data which would be very difficult or impossible to acquire with traditional scanning methods. Recent studies ${ }^{14,15}$ of the kinetics of annealing of strained epitaxial semiconductor layers illustrate another such application of CCDs. In this example we demonstrate the performance of a CCD as a fast streak camera. We have developed a new technique whereby a "rocking curve" scan can be recorded on very short time scale ( $\sim 15 \mu$ s currently) in order to probe the microscopic interface structure during rapid thermal annealing. It is necessary to use dynamical $x$-ray diffraction theory to analyze the resulting intensity profiles since the samples are close to the ideal crystal limit.

The key to being able to achieve such fast measurements here is to angularly disperse the synchrotron beam using an asymmetric-cut $\mathrm{Ge}(111)$ curved crystal monochromator. The fan of radiation incident on the sample is $\sim 250$ arcsec wide and is monochromatic to $\sim 2$ in $10^{4}$. Thus, using an area detector, each pixel records the scattered intensity at a particular angle of incidence, recording a whole rocking curve in one shot. Again, the absence of mechanical scanning allows for very fast data acquisition.

Figure 8 illustrates results from a recent study of a GaAs-InGaAs-GaAs single quantum-well (SQW) structure undergoing rapid thermal annealing. This thermal process is often used to improve the structural coherence of heterostructures since they are typically grown at relatively low temperatures where the kinetics are sluggish. Postgrowth annealing is also commonly used to activate ionimplanted dopants. However in all these post-growth thermal treatments very little is understood about the transient behavior of the structure. The time-resolved measurements presented here, which are the first of their kind, provide insight into the evolution of the interface structure as it approaches equilibrium. As clearly seen in Fig. 8, the annealing process does not proceed in a smooth continuous fashion. On the contrary, strongly discontinuous behavior is observed, revealing that the structure passes through several metastable states of strain on its way to equilibrium, which in this case is a perfectly coherent (pseudomorphic) arrangement of the three SQW layers. Further details on this application of CCDs can be found in a companion paper ${ }^{16}$ at this conference and also in Refs. 14 and 15 .

\section{FUTURE PROSPECTS AT THIRD GENERATION SOURCES}

For many applications, CCD pixel arrays have already proved to be a significant step forward beyond conventional single-channel $\mathrm{x}$-ray scintillation detectors; however CCDs may not satisfy the most demanding requirements associated with the high flux and high photon-energy characteristics which are being promoted at the European Synchrotron Radiation Facility (ESRF) and APS. For this reason, the quest for appropriate detector technology for third-generation sources should not be narrowly restricted at this stage.

The applications described here depend to a large extent on the efficient matching of $x$-ray optics to a fast parallel data acquisition method based on CCD pixel arrays. The imaging experiments are mostly brightness limited and significant improvements would be expected if low-emittance sources were used. The most remarkable advances however will be in the time resolution. Our current timeresolved experiments are made possible on a bending magnet source by the high degree of structural perfection of the samples. This directs a large scattered intensity into a narrow range of reciprocal space allowing us to accumulate good counting statistics on short time scales. Looking to the future we could envision much faster studies utilizing the pulsed structure of the synchrotron beam. The synchronization of the CCD row transfer with the arrival time of a single bunch would be one interesting possibility in the near future. Laser processing, growth studies, ion implantation kinetics, are all important materials areas that are candidates for real-time investigations at the new synchrotrons under construction. New developments in high resolution x-ray optics and fast pixel arrays will undoubtedly play a key role in the utilization of these specialized facilities.

\section{ACKNOWLEDGMENTS}

Work at the University of Michigan was supported in part by the National Science Foundation Grant No. 
DM8805165. The time-resolved synchrotron studies carried out at the National Synchrotron Light Source were performed on AT\&T Bell Laboratories' beamline X-16B. The NSLS is supported by the U.S.DoE, Basic Energy Science, Materials and Chemical Sciences, under Contract No. DE-AC02-76CA0016.

'Argonne Workshop on Time-resolved Studies and Ultrafast Detectors, Argonne, 1988, Report No. ANL/APS-TM-2.

${ }^{2}$ For a review see, B. C. Larson and J. Z. Tischler, SPIE 1345, 90 (1990).

${ }^{3}$ B. Rodricks, R. Clarke, R. Smither, and A. Fontaine, Rev. Sci. Instrum. 60, 2585 (1989).

${ }^{4} \mathrm{R}$. Clarke, Nucl. Instrum. Methods A 291, 117 (1990).

'S. M. Gruner, Science 238, 305 (1987); N. M. Allinson, R. Brammer, J.R. Helliwell, S. Harrop, B. G. Magorrian, and T. Wan, J. X-ray Sci. Technol. 1, 143 (1989); M. G. Strauss, I. Naday, I. S. Sherman, M. R. Kraimer, E. M. Westbrook, and N. J. Zaluzac, Nucl. Instrum. Methods A 266, 563 (1988).

${ }^{\circ}$ R. D. McGrath, IEEE Nucl. Sci. NS-28, 4028 (1981).
${ }^{7}$ R. MacHarrie, W. Lowe, and R. Clarke (to be published).

${ }^{8}$ M. P. Ulmer, R. Haidle, R. Altkorn, P. Georgopoulos, B. Rodricks, and P. Z. Takacs, Opt. Eng. (in press).

${ }^{9}$ P. F. Teaugue, M. P. Ulmer, Y. Matsui, U. G. Briel, W. Burkert, H. Bräuninger, and C. J. Hailey, Proc. SPIE 1160, 481 (1989).

${ }^{10}$ Y. Cauchois, CNRS Report 5 (1948).

"A. Fontaine, E. Dartyge, J. P. Itie, A. Jucha, A. Polian, H. Tolentino, and G. Tourillon, Mat. Res. Soc. Symp. Proc. 143, 121 (1989).

${ }^{12}$ B. Rodricks, F. Lamelas, D. Medjahed, W. Dos Passos, R. Smither, E. Ziegler, A. Fontaine, and R. Clarke, Mat. Res. Soc. Symp. Proc. 143, 209 (1989).

${ }^{13} \mathrm{~J}$. C. Campuzano, G. Jennings, L. Beaulaigue, B. G. Rodricks, and C. Brizard, SPIE 1345, 245 (1990).

${ }^{14}$ R. Clarke, W. Dos Passos, W. Lowe, B. G. Rodricks, and C. Brizard, Phys. Rev. Lett. 66, 317 (1991).

${ }^{15}$ W. Lowe, R. MacHarrie, R. Clarke, C. Brizard, and B. Rodricks, Phys. Rev. (to be published).

${ }^{16}$ W. Lowe, R. Clarke, W. Dos Passos, B. Rodricks, and C. Brizard, these proceedings. 